\title{
Electromyography in a Patient with Idiopathic Bruxism Treated with Occlusal Splint and Application of Botulinum Toxin- Case Report
}

\author{
Carrillo Rivera J1*, Villagrán RA², García LD³, Flores LR³ , González \\ Masso $\mathrm{JG}^{3}$, Inzunza $\mathrm{EH}^{3}$, Aguirre $\mathrm{SB}^{3}$, Juarez $\mathrm{MJ}^{4}$, Caltenco Solis \\ RB $^{5}$, González López ${ }^{5}$ and Ferrari V6 \\ ${ }^{1}$ Stomatologist, Oral and Maxillofacial Surgery, General Hospital "Dr. Darío Fernández \\ Fierro "ISSSTE, Professor in Justo Sierra University, Private practice, Mexico
}

\section{Research Article}

Volume 2 Issue 2

Received Date: July 26, 2018

Published Date: August 17, 2018

DOI: $10.23880 /$ ijtps-16000121

2Stomatologist, Oral Prosthesis and Rehabilitation, Postgraduate professor of oral prosthesis, Universidad Latinoamericana, Private practice Mexico

${ }^{3}$ Dental Surgeon, Oral and Maxillofacial Surgery, Private practice, Mexico

${ }^{4}$ Dental Surgeon, Maxillofacial prosthesis, School of Higher Studies Iztacala, UNAM, Justo Sierra University, Vice-president of the Mexican school of maxillofacial prostheses and oncological stomatology, Private practice, Mexico

${ }^{5}$ Second-year resident in General Surgery, General Hospital Darío Fernández Fierro, ISSST, Mexico

${ }^{6}$ Biomedical engineer, Milano, Italy

*Corresponding author: Dr. Jorge Arnulfo Carrillo Rivera, Dental and Maxillofacial Rehabilitation, Office 124, Juárez Avenue, Number 8, Colonia Las Margaritas, Tlalnepantla State of Mexico, Tel: 53618677; Email: jcarrillo_99@yahoo.com

\section{Abstract}

Electromyography is a study that allows evaluating the motor and sensory function of the neuromuscular system through the recording of the electrical stimulus.

There are few studies related to electromyography in patients with idiopathic or primary bruxism. The aim of the present study was to evaluate the electromyographic activity of the masseter and temporal muscles in a patient with primary bruxism before and after the use of the occlusal splint and the application of botulinum toxin.

Keywords: Electromyography; Bruxism; Occlusal splint; Botulinum toxin

\section{Introduction}

Electrophysiology began in the late eighteenth century with the discovery of animal electricity by GALVANI, which achieved muscle contraction by stimulating the free termination of a nerve, without using metals for it; Humbold in 1797 and Marreuccí reproduced these findings in 1844. Duchenne de Eculogne, in 1833 saw that a muscle could be stimulated electrically from the skin surface by means of electrodes; it was also the first to use 


\section{International Journal of Transplantation \& Plastic Surgery}

the current for stimulation [1]. In 1922, Gasser and Erlanger contributed one of the most important advances in technology, the cathode ray oscilloscope that eliminated the mechanical limitations of galvanometers. At the same time, there was a new advance in neurophysiological knowledge, since Hoffmann in 1922 discovered the monosynaptic reflexes in man stimulating the tibial nerve and recording the action potential of the soleus muscle [2].

The contractile unit of the musculature of the skeleton is the muscle fiber, which is a cylindrical cell about $50 \mu \mathrm{m}$ in diameter, which, when stimulated, contracts to develop a force. A muscle consists of parallel bundles of muscle fibers. The activation of each muscle fiber is done through the axon of the motor nerve fiber that innervates it. Depending on the position and function of the muscle, the number of muscle fibers innervated by the same axon can vary between one or more than a thousand. The group formed by the motor nerve cell in the spine, its axon and the muscle fibers that it innervates constitutes the basic functional unit of the muscular system and is known as the motor unit. When the action potential of the nerve reaches the point at which it joins the muscle, a certain amount of a chemical transmitter (acetylcholine) is released, which causes local depolarization of the muscle fiber membrane. The transmitter is quickly neutralized by a substance called acetylcholine estereate, leaving the myoneuronal junction free for a new excitation $[1,2]$.

Currently electromyography is defined as a technique of recording the electrical signal that originates in the muscle fiber and is transmitted through adjacent tissues that allows recording muscle activity during the different functions performed by that muscle [3].

One of the objectives of electromyography is to evaluate the motor and sensory function of the neuromuscular system by measuring the electrical stimulus observed in an electromagnetic wave; This search for the relationship between the shape and function of the muscular apparatus has led to an increase in its use as a tool for research in quantitative oral physiology $[1,2]$.

The electromyographic analysis allows measuring the electrical activity of the large chewing muscles and all the information obtained is used to evaluate the occlusal plane from the neuromuscular point of view.

Bruxism is defined as the parafuncional habit diurnal or nocturnal that consists of tightening or grinding the dental organs voluntarily and involuntarily and of variable etiology. To assess bruxism, a detailed clinical examination should be performed integrating the morphological part (which is usually analyzed in the physical examination) and the functional part evaluated by means of electromyography.

The electromyography allows knowing if the prevalence of occlusal contact is greater in the premolars or molars, on the right or left side, if there is or not a torsional behavior of the jaw and if the work produced in the maximum voluntary contraction force is high or low.

Electromyography in patients with bruxism, also allows us to know the prevalence of tightening, and suggests the type of occlusal guard to be used, decreasing the contact area (or increasing the contralateral area) to achieve a balanced occlusion, in addition, it determines the use of botulinum toxin In the hypertonic masticatory muscle to limit the maximum contraction force, compare the muscular strength of both sides of the face to know if there is a determining side, and evaluate the lateral displacement of the jaw or torsion in the horizontal plane.

\section{Clinical Case}

A 62-year-old female patient without systemic diseases with a clenching and bruxism habit accompanied by pain on the EVA scale 8 out of 10 , in the right hemifacial region of 5 years of evolution treated with analgesics, non-steroidal anti-inflammatory drugs and rigid occlusal guard without improvement (Figure 1).

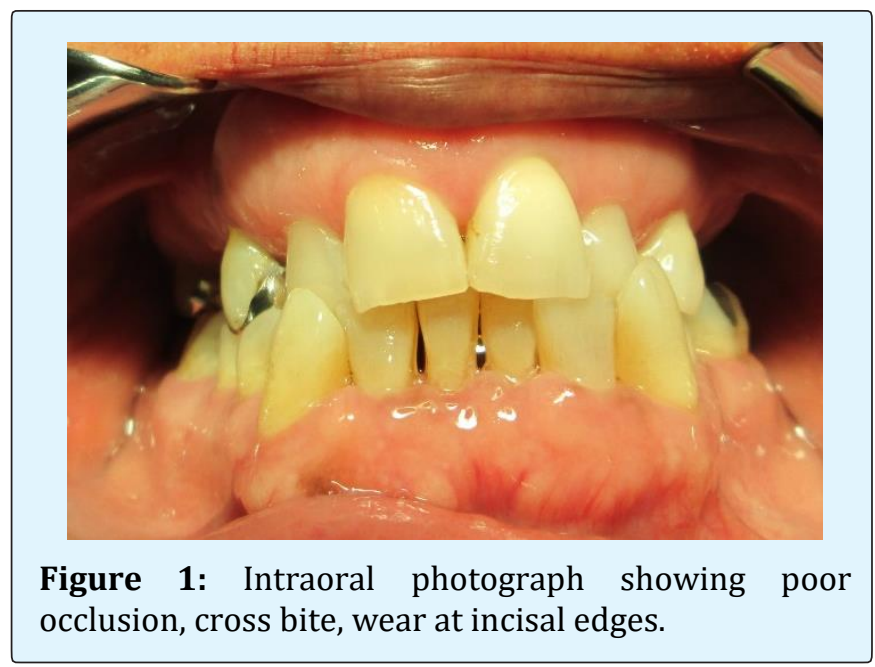

The general data were obtained by means of the interrogatory recording in the formats of examination of 


\section{International Journal of Transplantation \& Plastic Surgery}

the clinical characteristics and in the questionnaires according to the recommendations of the diagnostic criteria of the disorders of the temporomandibular joint (DC/TMD). Teethan ${ }^{\circledR}$ surface electromyography was performed to evaluate the intensity of the contraction of each muscle. The bar graph mentions the muscle with the highest activity (Figure 2) [4].
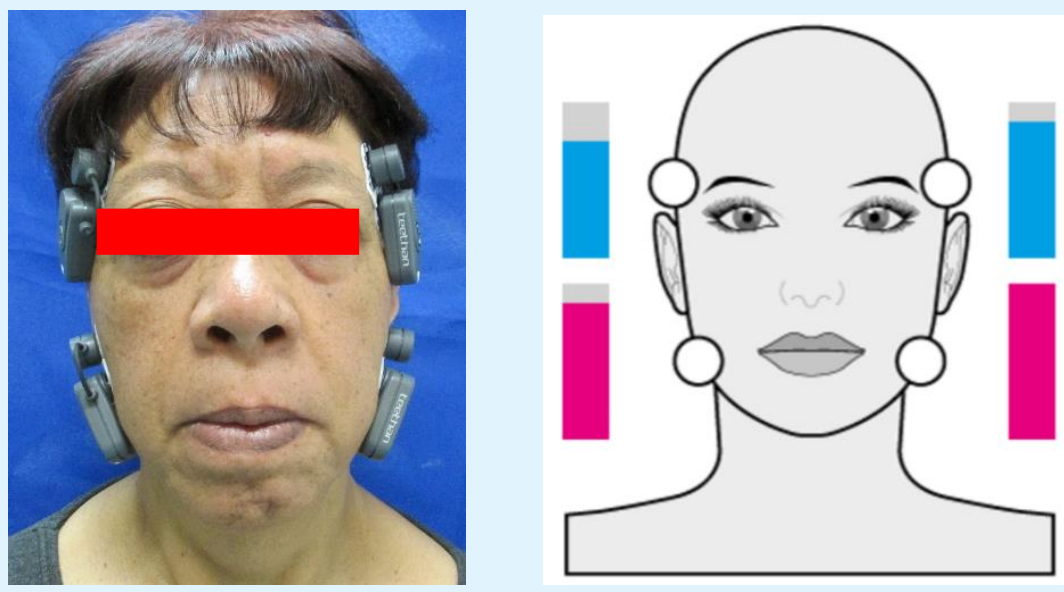

Figure 2: Anteroposterior photograph showing the placement of the surface electrodes. Bar graph showing greater activity in left temporal and masseter muscles.

The maximum contraction force determined in the electromyography was high in the left masseter muscle followed by the left temporalis muscle, right masseter and right temporalis muscle, the left hemisphere being predominant and with a greater degree of torsion towards the right side of the jaw, with a prevalence of occlusal contact in the left premolars. The application of botulinum toxin was decided with a dilution of 1 to 1 in physiological solution and applying 5 international units in three anatomical points in the left masseter muscle and two anatomical points in the left temporal bone (Figure $3)$.
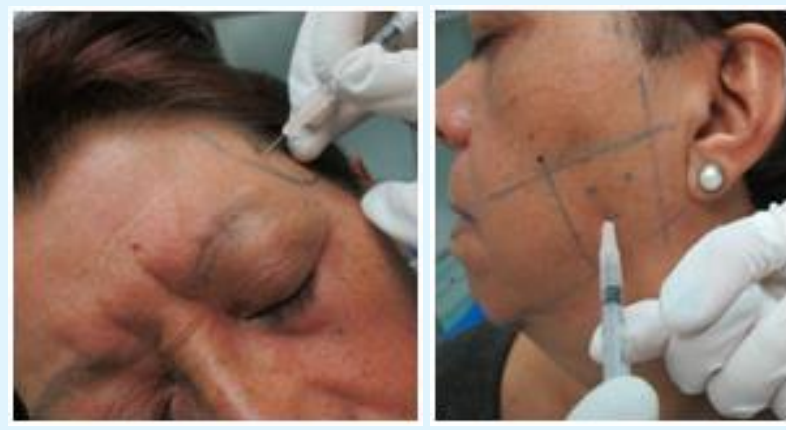

Figure 3: Application of botulinum toxin in left masseter and temporal muscle.
An acrylic occlusal splint was performed with an incisal table to decrease contact in the premolars and molars for three weeks and then to start the definitive occlusal treatment (Figure 4).

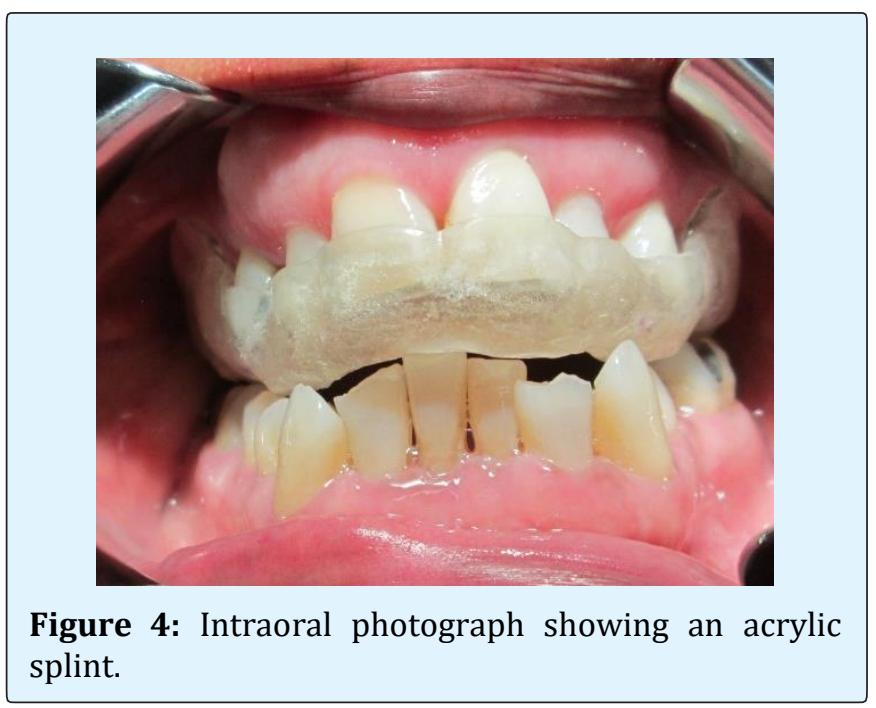

Control was cited by reviewing clinically anatomical points, referring improvement to painful symptoms on EVA scale 2 out of 10 . Control electromyography was performed, appreciating improvement to the clenching habit with more balanced maximum contraction indices 


\section{International Journal of Transplantation \& Plastic Surgery}

and decrease in excessive force in left hemisphere, as well as improvement to the degree of mandibular torsion (Figures 5 \& 6).

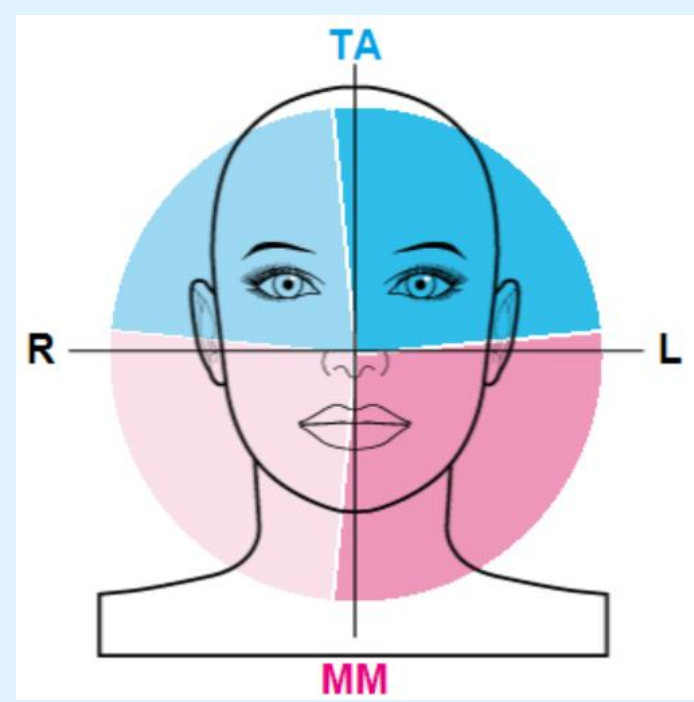

Figure 5: Electromyography of pie chart control where muscle activity is observed in the four quadrants after the infiltration of botulinum toxin.

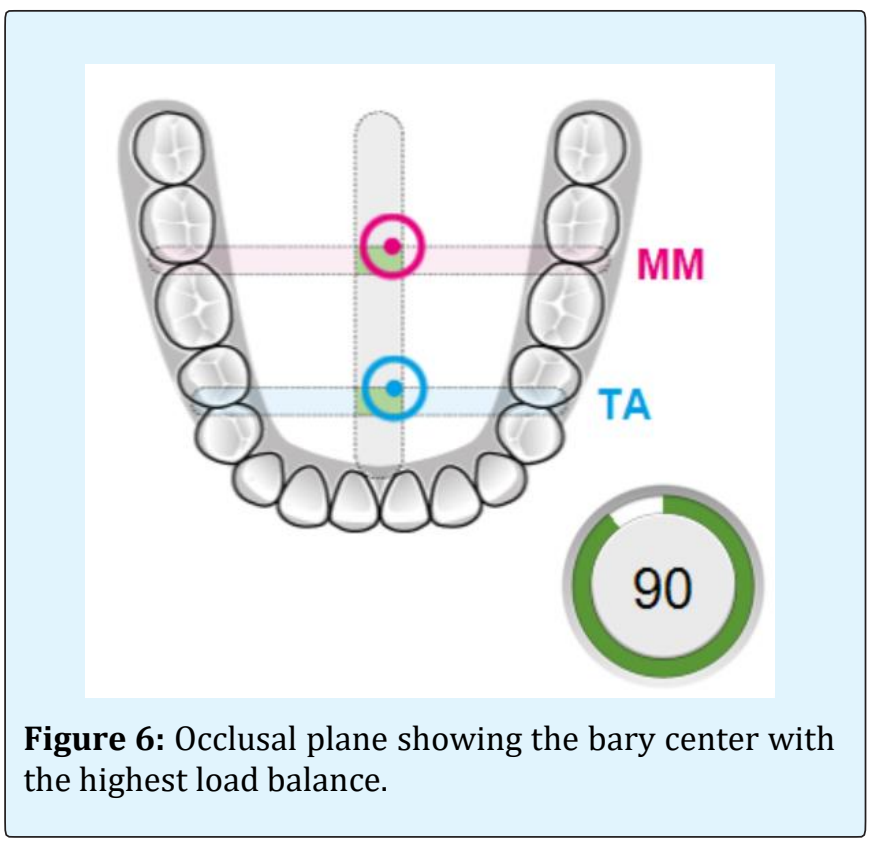

\section{Results}

According to the results obtained, electromyography allows to be a guide in the treatment of bruxism when determining the excessive muscular strength in the masticatory muscles and to focus the treatment when balancing the occlusal plane. In the present case, the pain was reported in the right side of the face, however when performing the electromyography, the prevalence of occlusal contact and the maximum contraction force was in the left side of the face, with a greater torsional torque of the mandible towards the right side, so the application of botulinum toxin was on the left side, achieving a balance in the occlusal loads and improving the tightening with the use of an occlusal splint made with precise specifications for the patient.

\section{Discussion}

For more than 20 years, multiple studies of the validity and reliability of the EMG have been carried out. Currently, its use is being taught in 40 universities around the world with more than 120 studies carried out. The electromyographic findings found about muscle activity mention that patients with functional temporomandibular disorders tend to have muscle atrophy unlike healthy asymptomatic patients. This muscle dysfunction can be measured by means of EMG, knowing its severity and giving treatment successfully.

Frequently, the treatment mentioned for the bruxism in different articles, includes the occlusal adjustment, eliminating premature contact points and the use of a rigid occlusal guard to avoid wear on the dental organs and improve the vertical dimension, however these treatments have a limit therapeutic because they do not resolve the muscle tightening habit, in addition to the nociceptive reflex that may be present.

The treatment includes the use of restrictive and nonrestrictive occlusal splints, in a therapeutic but not resolutive way, the opportune diagnosis by means of the physical examination and the electromyography allows to know the physiological condition, giving a specific treatment in the hypertonic muscles.

\section{Conclusion}

Electromyography (EMG) is the study of the electrical activity of the muscles, which quantitatively measures and documents their functionality, as well as the severity and progression of muscular ailments, helping to guide the treatment of temporomandibular disorders.

Bruxism is a parafuncional activity that conditions the tightening and sliding of the dental organs, this condition 


\section{International Journal of Transplantation \& Plastic Surgery}

is multifactorial but it has been related to the excessive force of one or several chewing muscles, if the prevalence of occlusal contacts is constant it can cause headache, painful syndrome myofascial and lead to long-term joint pathologies.

The excessive tightening force conditions the muscular hypertonicity and varies in each patient; therefore, the application of botulinum toxin should not be applied in all four muscles.

The intensity of maximum contraction is evaluated by means of surface electromyography in the masseter and temporal muscles, appreciating their activity.

When applying botulinum toxin in the masseter and temporal muscles, a functional change will be obtained, either to increase or decrease the contraction and therefore the strength.

Except for a muscular disease, the muscles act depending on the structure below (the occlusal plane) and immediately react to the lack / excess of contact surface.

\section{Ethical Aspects}

This study was carried out in accordance with the Declaration of Helsinki and the provisions of the General Health Law regarding research on Human Beings in Mexico.
According to the General Health Law, considering Art. 3, 14 and 17 that includes it within the category: Research with minimal risk, because they will use common procedures such as anthropometric measurements, biochemical measurements and diet treatment. -Routine therapeutic.

Likewise, based on Art 22 of the same Law, informed consent will be requested in writing to the patient.

\section{References}

1. Caballero K, Duque LM, Ceballos S, Ramírez JC, Peláez A (2002) Basic concepts of electromyographic analysis. Rev CES Odont 15(1): 41-50.

2. Barraquer-Bordas L (1963) Fundamental Neurology. Barcelona: Ed. Toray SA, pp: 502-567.

3. Michael J Aminoff (2012) Clinical Electromyography, Aminoff's Electrodiagnosis in Clinical Neurology. $6^{\text {th }}$ (Edn.), San Francisco, CA, Elsevier.

4. Tartaglia GM, Lodetti G, Paiva G, Felício CM, Sforza C (2011) Surface electromyography assessment of patients with long lasting temporomandibular joint disorder pain. J Electromyogr Kinesiol 21: 659-664. 